\title{
Association between expression of DNA mismatch repair genes and clinical features and prognosis of patients with radical resection of colon cancer
}

\author{
J.B. Wang ${ }^{1 *}$, D.L. Ma ${ }^{2 *}$, J.Y. Li ${ }^{2}$, Q.D. Sun ${ }^{1}$ and Y.E. Liu $^{3}$ \\ 'Department of General Surgery, Yishui Central Hospital of Linyi, \\ Linyi, Shandong, China \\ ${ }^{2}$ Department of Medical Oncology, Yishui Central Hospital of Linyi, \\ Linyi, Shandong, China \\ ${ }^{3}$ Department of Infectious Diseases, Yishui Central Hospital of Linyi, \\ Linyi, Shandong, China \\ *These authors contributed equally to this study. \\ Corresponding author: Y.E. Liu \\ E-mail:2369475216@qq.com
}

Genet. Mol. Res. 15 (3): gmr. 15038388

Received January 7, 2016

Accepted March 28, 2016

Published August 18, 2016

DOI http://dx.doi.org/10.4238/gmr.15038388

Copyright (C) 2016 The Authors. This is an open-access article distributed under the terms of the Creative Commons Attribution ShareAlike (CC BY-SA) 4.0 License.

\begin{abstract}
The aim of this study was to investigate the clinical significance of the expression of DNA mismatch repair (MMR) genes in patients subjected to radical surgical removal of colon cancer, as well as their correlation with disease prognosis. Ninety stage II and III colon cancer patients who received laparoscopic radical resection of colon cancer at our hospital were recruited in this study. The expression of hMLH1, hMSH2, hMSH6, and hPMS2 in the resected tumor tissues was examined by SP immunohistochemistry, in order to analyze the
\end{abstract}


relationship between defective DNA MMR (dMMR) and the clinicopathological features and prognosis of colon cancer. Patients were followed up over a period of 5-35 months, and the Kaplan-Meier survival curve was plotted. dMMR was confirmed in 27 subjects $(30.0 \%)$, among whom recurrence with metastasis and death was reported in 5 (18.5\%) and $2(7.4 \%)$ patients, respectively. The remaining 63 subjects displayed proficient DNA MMR (pMMR); among these, $19(30.2 \%)$ and $7(11.1 \%)$ recurrences with metastasis and death were reported, respectively. dMMR showed no significant correlation with gender, age, or therapeutic modality $(\mathrm{P}>0.05)$, but was significantly correlated with the degree of differentiation, tumor location, number of resected lymph nodes, presence of ileus, and TNM stage $(\mathrm{P}<0.05)$. The prognosis of patients with dMMR was better than that of patients with pMMR. dMMR serves as a biomarker for the prognosis of stage II/III colon cancers.

Key words: Colon cancer; Radical resection of colon cancer; DNA mismatch repair gene; Prognosis

\section{INTRODUCTION}

Colon cancer is one of the most common digestive tract cancers, and has the third highest prevalence among all cancers. Surgical resection is the current major treatment for colon cancer. However, as tiny lesions may be overlooked during the surgery, the possibility of recurrence in patients with stage II/III colon cancers is approximately 20-50\% (Clarizia et al., 2006; De Sousa E. Melo et al., 2013; Jin et al., 2014; Parnaby et al., 2015; Sun et al., 2015; Tan et al., 2015). DNA mismatch repair (MMR) proteins, such as hMLH1, hMSH2, hMSH6, and hPMS2, correct base mismatches that occur during DNA replication, and are therefore an important component in maintaining genomic stability (Erie and Weninger, 2014; Xu et al., 2015). Defective DNA MMR (dMMR) has been implicated in $15 \%$ of all colon cancer cases (Sinicrope et al., 2011; Haraldsdottir et al., 2015). Therefore, the aim of this study was to investigate the clinical significance of the expression of MMR genes in patients with surgical resection of colon cancer.

\section{MATERIAL AND METHODS}

\section{Patients}

Ninety stage II or III colon cancer patients (58 males and 32 females; mean age, 62.19 \pm 7.28 years) who underwent a laparoscopic radical resection of colon cancer at our hospital between May 2011 and May 2012 were recruited to this study. Colon cancer was categorized into stages II or III according to the staging criteria set by the American Joint Committee on Cancer (AJCC) (7th edition). Patient follow-ups were performed by visits to the clinic or by telephone until December 1, 2014. This study was approved by the Ethics Committee at the Yishui Central Hospital of Linyi, and signed informed consent was obtained from all patients prior to this study.

Genetics and Molecular Research 15 (3): gmr.15038388 


\section{Detection of DNA MMR state by immunohistochemistry}

The expression levels of hMLH1, hMSH2, hMSH6, and hPMS2 were determined by SP immunohistochemistry. Paraffin-embedded sections were prepared from $>50 \%$ of the tumor area in the tissue sample resected from patients during surgery. The sections were dried in a $60^{\circ} \mathrm{C}$ incubator, dewaxed in xylene, and dehydrated in gradient ethanol. Antigens were retrieved from the sections using $1 \mathrm{M}$ EDTA, $\mathrm{pH}$ 8.0. The sections were then incubated in 3\% $\mathrm{H}_{2} \mathrm{O}_{2}$ for $10 \mathrm{~min}$, washed thrice in phosphate-buffered saline (PBS) for $5 \mathrm{~min}$ each, incubated with goat serum for $15 \mathrm{~min}$, and subsequently incubated again with primary antibodies against hMLH1, hMSH2, hMSH6, or hPMS2 (Dako, Glostrup, Denmark) for $3 \mathrm{~h}$ at $37^{\circ} \mathrm{C}$. The sections were washed thrice with PBS ( 5 min each), both before and after incubation with a biotin-labeled goat anti-mouse IgG for $20 \mathrm{~min}$ at $37^{\circ} \mathrm{C}$. The sections were then incubated with horseradish peroxidase-conjugated streptavidin for $15 \mathrm{~min}$ at $30^{\circ} \mathrm{C}$ followed by a PBS washing step (thrice; 5 min each). The chromogenic reagent DAB was added to the sections, followed by regular counter-staining, washing, dehydration in gradient ethanol, and drying. The stained sections were observed under an optical microscope.

\section{Assessment criteria}

The average of 10 randomly selected microscopic fields was recorded. The sections were scored based on the staining intensity and number of positively stained cells, as follows: 0 , unstained; 1, light yellow; 2 , yellow, and 3, brown; and $0, \leq 10 \%$ positive cells; $1,11-30 \%$ positive cells; $2,31-70 \%$ positive cells; and 3, $>70 \%$ positive cells. The sections were classified based on the combined score: negative (-) for a combined score of 0-2 and positive (+) for a combined score of 3 and above. The particles stained a brownish color in hMLH1-, hMSH2-, hMSH6-, and hPMS2-positive cells were mainly located in the cell nucleus. Sections that did not express one or more of these proteins (hMLH1, hMSH2, hMSH6, and hPMS2) were denoted dMMR, while sections that were positive stained for all four proteins were denoted proficient DNA MMR (pMMR).

\section{Statistical analysis}

The data were analyzed using the SPSS 19.0 statistical software package (IBM, Armonk, NY, USA). All data are reported as means \pm standard deviation (SD) of multiple analyses; the differences were assessed by analysis of variance (ANOVA). A P value $<0.05$ indicated a statistically significant difference.

\section{RESULTS}

\section{Expression of MMR proteins}

The follow-up period lasted 5-35 months, with a median and average follow-up period of 27 and 25.48 months, respectively. Twenty-four patients showed relapse with metastasis during the follow-up period, whereas 9 patients died (including 1 who died from reasons other than tumor). dMMR was detected in 27 patients $(30 \%)$, with 5 recurrences with metastasis $(18.5 \%)$ and 2 deaths (7.4\%). Meanwhile, pMMR was detected in 63 patients (70\%), with

Genetics and Molecular Research 15 (3): gmr.15038388 
$19(30.2 \%)$ recurrences/metastasis and 7 deaths (11.1\%). Typical positive staining of MMR proteins is shown in Figure 1.

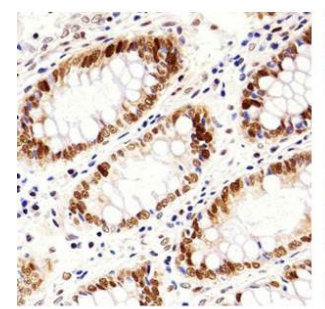

hMLH1

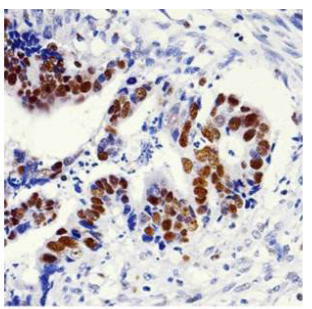

hMSH2

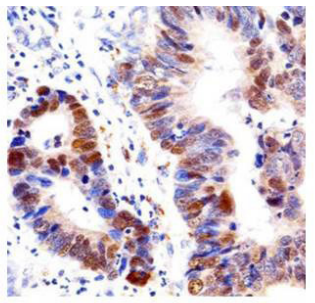

hMSH6

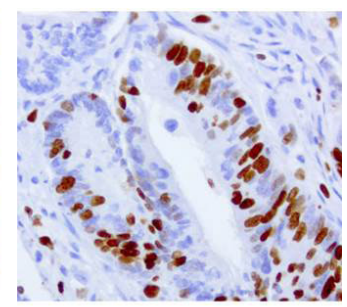

hPMS2

Figure 1. Positive expression of hMLH1, hMSH2, hMSH6, and hPMS2 (400X).

\section{Relationship between dMMR and clinical pathological features}

Analysis of the relationship between dMMR and the clinico-pathological features of colon cancer showed that dMMR was not significantly related to gender, age, or therapeutic modality $(\mathrm{P}>0.05)$. However, dMMR was significantly associated with the degree of differentiation, tumor location, number of resected lymph nodes, presence of ileus, and TNM stage $(\mathrm{P}<0.05)$. The results indicated that the probability of dMMR was higher in patients with poorly differentiated, proximal, stage II colon cancer without ileus, and in whom $>12$ lymph nodes were resected (Table 1).

Table 1. Relationship between deficient mismatch repair and clinico-pathological features of colon cancer.

\begin{tabular}{|c|c|c|c|c|c|}
\hline Clinical pathological features & Total cases $(\mathrm{N})$ & pMMR $(\mathrm{N})$ & dMMR (N) & $\chi^{2}$ value & $P$ value \\
\hline \multicolumn{6}{|l|}{ Gender } \\
\hline Male & 58 & 42 & 16 & \multirow[t]{2}{*}{0.453} & \multirow[t]{2}{*}{0.501} \\
\hline Female & 32 & 21 & 11 & & \\
\hline \multicolumn{6}{|l|}{ Age } \\
\hline$>65$ years & 33 & 22 & 11 & \multirow[t]{2}{*}{0.430} & \multirow[t]{2}{*}{0.512} \\
\hline$\leq 65$ years & 57 & 42 & 15 & & \\
\hline \multicolumn{6}{|l|}{ Therapeutic modality } \\
\hline Single treatment & 33 & 18 & 15 & \multirow[t]{2}{*}{2.423} & \multirow[t]{2}{*}{0.171} \\
\hline Post-surgical 5-FU/LV & 57 & 45 & 12 & & \\
\hline \multicolumn{6}{|l|}{ Degree of differentiation } \\
\hline Moderate-highly differentiated & 35 & 25 & 10 & \multirow[t]{2}{*}{6.080} & \multirow[t]{2}{*}{0.014} \\
\hline Poorly differentiated & 55 & 38 & 17 & & \\
\hline \multicolumn{6}{|l|}{ Tumor location } \\
\hline Proximal & 38 & 20 & 18 & \multirow[t]{2}{*}{4.592} & \multirow[t]{2}{*}{0.032} \\
\hline Distal & 52 & 39 & 13 & & \\
\hline \multicolumn{6}{|l|}{ Number of resected lymph nodes } \\
\hline$>12$ & 35 & 16 & 19 & \multirow[t]{2}{*}{4.870} & \multirow[t]{2}{*}{0.027} \\
\hline$\leq 12$ & 55 & 38 & 17 & & \\
\hline \multicolumn{6}{|l|}{ Presence of ileus } \\
\hline No & 44 & 27 & 17 & \multirow[t]{2}{*}{4.403} & \multirow[t]{2}{*}{0.036} \\
\hline Yes & 46 & 34 & 12 & & \\
\hline \multicolumn{6}{|l|}{ TNM stage } \\
\hline II & 48 & 30 & 18 & \multirow[t]{2}{*}{6.195} & \multirow[t]{2}{*}{0.013} \\
\hline III & 42 & 31 & 11 & & \\
\hline
\end{tabular}

pMMR, proficient DNA mismatch repair; dMMR, deficient DNA mismatch repair.

Genetics and Molecular Research 15 (3): gmr.15038388 


\section{Relationship between dMMR and prognosis}

The Kaplan-Meier survival curves of patients with dMMR or pMMR indicated better prognosis in patients with dMMR compared to that in patients with pMMR ( $\mathrm{P}=0.0007$; Figure 2), suggesting that dMMR is a good biomarker for better prognosis.

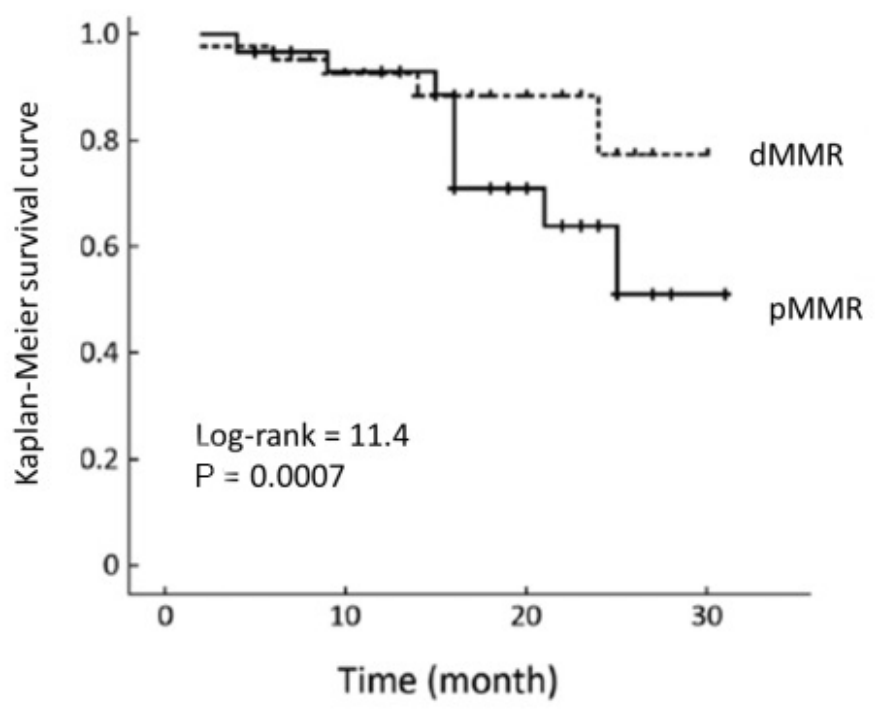

Figure 2. Kaplan-Meier survival curve.

\section{DISCUSSION}

Colon cancer is one of the most common types of malignant cancers, with an increasing incidence in China. Radical therapeutic strategies for colon cancer include laparoscopic resection and open resection. In particular, laparoscopic radical resection has several advantages, including minimal invasion, rapid post-operative recovery, less bleeding, and fewer complications (Mörner et al., 2012). All subjects enrolled in this study underwent laparoscopic radical resection of colon cancer, thereby minimizing the potential impact of radical resectionassociated complications and adverse effects on data interpretation. The 5-year survival rate of colon cancer in China is approximately $31 \%$; moreover, the main cause of colon cancerrelated death is recurrence because of tiny lesions that are overlooked during surgical resection. Previous studies have shown that approximately $85 \%$ of focal lesions in colon cancer display chromosomal instability, a critical reason for the pathogenesis and poor prognosis of colon cancer. Nearly $15 \%$ of all patients with sporadic colon cancer present dMMR (Pogue-Geile et al., 2013; Lamba et al., 2015). Therefore, the aim of this study was to explore the significance of dMMR in the clinico-pathological features and prognosis of colon cancer.

The DNA MMR system is involved in repairing mismatched bases missed by the DNA polymerase proofreading activity during DNA replication, in order to maintain the stability of the genome and prevent cell transformation and oncogenesis resulting from genetic mutation. Key players in the DNA MMR system include hMLH1, hMSH2, hMSH6, and hPMS2. Other

Genetics and Molecular Research 15 (3): gmr.15038388 
MMR proteins include hMLH3, hMSH3, hPMS1, and hPMS3. Mutations in hMLH1, as well hMSH2, account for $40-50 \%$ of dMMR; on the other hand, the hMSH6 mutation accounted for $5-10 \%$ of dMMR, whereas the hPMS2 mutation only accounted for $1 \%$. hMSH2 and hMSH6 form a heterodimer during DNA replication that binds to mismatched bases and interacts with the hMLH1/hMSH2 heterodimer. The repair reaction is initiated by excising the DNA fragment containing the mismatched base, followed by a polymerase-mediated replication of the excised oligonucleotides and ligation of the repaired DNA. dMMR is primarily caused by mutations in the hMLH1, hMSH2, hMSH6, and hPMS2 genes, or as a result of DNA methylation (Hombauer et al., 2011). In this study, we assessed the expression levels of hMLH1, hMSH2, hMSH6, and hPMS2 in colon cancer tissues, and showed the presence of dMMR and pMMR in $27(30 \%)$ and $63(70 \%)$ patients, respectively.

Stage II colon cancer is the invasion of cancer cells to all layers of the bowel wall without lymph node or distant metastasis, which is characteristic of stage III colon cancer. A previous study reported that the incidence rate of dMMR was 13.1-30.6\% in stage II/III colon cancer patients; moreover, post-operative mortality in patients with dMMR was up to $50 \%$, suggesting that MMR function may be associated with the clinical features and prognosis of, and high post-operative recurrence in, stage II/III colon cancers (McCleary et al., 2013; Olsen et al., 2016). The results of these studies influenced the enrollment of stage II and III colon cancer patients. Analysis of the relationship between MMR function and the clinical features of the colon cancer patients revealed that dMMR was not correlated with gender, age, or therapeutic modality $(\mathrm{P}>0.05)$. In clinical practice, patients are usually treated with 5-FU/LV for 6 months after radical resection of colon cancer to consolidate the therapeutic effect; the effect of 5-FU is based on stable proofreading of the DNA MMR system (Akao et al., 2011). Therefore, patients may develop resistance to 5-FU treatment in the presence of dMMR. In this study, the rate of dMMR was not significantly different between patients treated with 5-FU/LV and those that received single radical surgery, implying that MMR function did not enhance the therapeutic effect of 5-FU/LV. In contrast, the rate of dMMR was significantly different $(\mathrm{P}<0.05)$ between patients with different degrees of differentiation, tumor location, number of resected lymph nodes, TNM stages, and presence/absence of ileus. The frequency of dMMR was relatively higher in patients with poorly differentiated, or stage II, colon cancer, whereas the frequency was relatively lower when the tumor was in a distal location or in the presence of ileus. The frequency of dMMR was higher in patients with $>12$ resected lymph nodes compared to those with $\leq 12$ resected lymph nodes.

During the follow-up period, 5 cases $(18.5 \%)$ of recurrence with metastasis and 2 (7.4\%) deaths were reported in patients with dMMR, whereas 19 cases $(30.2 \%)$ of recurrence with metastasis and 7 deaths $(11.1 \%)$ were reported in patients with pMMR. The KaplanMeier survival curve showed that the prognosis of patients with dMMR was significantly better than that of patients with pMMR $(\mathrm{P}=0.0007)$. These results suggest that dMMR is a good biomarker for better prognosis, which is consistent with the findings of previous studies. Sargent et al. (2010) reported a high 5-year recurrence-free rate (80\%) in colon cancer patients with dMMR who received a single surgical treatment (56\% higher than those with pMMR). The 5-year survival rate was also significantly higher in patients with dMMR than in those with pMMR (Sargent et al., 2010). In addition, Hutchins et al. (2011) reported a markedly lower post-surgical recurrence rate in colon cancer patients with dMMR compared to that in patients with pMMR.

In conclusion, our study shows that dMMR often occurs in patients with poorly

Genetics and Molecular Research 15 (3): gmr.15038388 
differentiated and low-stage colon cancer, which suggests that dMMR can serve as a biomarker for the prognosis of stage II/III colon cancers.

\section{Conflicts of interest}

The authors declare no conflict of interest.

\section{ACKNOWLEDGMENTS}

The authors thank all the patients and family members for their participation. Research supported by the Yishui Central Hospital of Linyi.

\section{REFERENCES}

Akao Y, Noguchi S, Iio A, Kojima K, et al. (2011). Dysregulation of microRNA-34a expression causes drug-resistance to 5-FU in human colon cancer DLD-1 cells. Cancer Lett. 300: 197-204. http://dx.doi.org/10.1016/j.canlet.2010.10.006

Clarizia AD, Bastos-Rodrigues L, Pena HB, Anacleto C, et al. (2006). Relationship of the methylenetetrahydrofolate reductase C677T polymorphism with microsatellite instability and promoter hypermethylation in sporadic colorectal cancer. Genet. Mol. Res. 5: 315-322.

De Sousa E Melo F, Wang X, Jansen M, Fessler E, et al. (2013). Poor-prognosis colon cancer is defined by a molecularly distinct subtype and develops from serrated precursor lesions. Nat. Med. 19: 614-618. http://dx.doi.org/10.1038/ $\underline{\mathrm{nm} .3174}$

Erie DA and Weninger KR (2014). Single molecule studies of DNA mismatch repair. DNA Repair 20: 71-81. http://dx.doi. org/10.1016/j.dnarep.2014.03.007

Haraldsdottir S, Roth R, Pearlman R, Hampel H, et al. (2015). Mismatch repair deficiency concordance between primary colorectal cancer and corresponding metastasis. Fam. Cancer 14: 1-8.

Hombauer H, Campbell CS, Smith CE, Desai A, et al. (2011). Visualization of eukaryotic DNA mismatch repair reveals distinct recognition and repair intermediates. Cell 147: 1040-1053. http://dx.doi.org/10.1016/j.cell.2011.10.025

Hutchins G, Southward K, Handley K, Magill L, et al. (2011). Value of mismatch repair, KRAS, and BRAF mutations in predicting recurrence and benefits from chemotherapy in colorectal cancer. J. Clin. Oncol. 29: 1261-1270. http:// dx.doi.org/10.1200/JCO.2010.30.1366

Jin J, Xie L, Xie CH and Zhou YF (2014). Aberrant DNA methylation of MGMT and hMLH1 genes in prediction of gastric cancer. Genet. Mol. Res. 13: 4140-4145. http://dx.doi.org/10.4238/2014.May.30.9

Lamba AR, Moore AY, Moore T, Rhees J, et al. (2015). Defective DNA mismatch repair activity is common in sebaceous neoplasms, and may be an ineffective approach to screen for Lynch syndrome. Fam. Cancer 14: 259-264. http:// dx.doi.org/10.1007/s10689-015-9782-3

McCleary NJ, Meyerhardt JA, Green E, Yothers G, et al. (2013). Impact of age on the efficacy of newer adjuvant therapies in patients with stage II/III colon cancer: findings from the ACCENT database. J. Clin. Oncol. 31: 2600-2606. http:// dx.doi.org/10.1200/JCO.2013.49.6638

Mörner ME, Gunnarsson U, Jestin P and Svanfeldt M (2012). The importance of blood loss during colon cancer surgery for long-term survival: an epidemiological study based on a population based register. Ann. Surg. 255: 1126-1128. http://dx.doi.org/10.1097/SLA.0b013e3182512df0

Olsen J, Eiholm S, Kirkeby LT, Espersen ML, et al. (2016). CDX2 downregulation is associated with poor differentiation and MMR deficiency in colon cancer. Exp. Mol. Pathol. 100: 59-66. http://dx.doi.org/10.1016/j.yexmp.2015.11.009

Parnaby CN, Scott NW, Ramsay G, MacKay C, et al. (2015). Prognostic value of lymph node ratio and extramural vascular invasion on survival for patients undergoing curative colon cancer resection. Br. J. Cancer 113: 212-219. http://dx.doi.org/10.1038/bjc.2015.211

Pogue-Geile K, Yothers G, Taniyama Y, Tanaka N, et al. (2013). Defective mismatch repair and benefit from bevacizumab for colon cancer: findings from NSABP C-08. J. Natl. Cancer Inst. 105: 989-992. http://dx.doi.org/10.1093/jnci/djt140

Sargent DJ, Marsoni S, Monges G, Thibodeau SN, et al. (2010). Defective mismatch repair as a predictive marker for lack of efficacy of fluorouracil-based adjuvant therapy in colon cancer. J. Clin. Oncol. 28: 3219-3226. http://dx.doi. org/10.1200/JCO.2009.27.1825

Genetics and Molecular Research 15 (3): gmr.15038388 
Sinicrope FA, Foster NR, Thibodeau SN, Marsoni S, et al. (2011). DNA mismatch repair status and colon cancer recurrence and survival in clinical trials of 5-fluorouracil-based adjuvant therapy. J. Natl. Cancer Inst. 103: 863-875. http://dx.doi.org/10.1093/jnci/djr153

Sun L, Li X, Li Z, Li Z, et al. (2015). Improving antitumor activity with N-trimethyl chitosan entrapping camptothecin in colon cancer and lung cancer. J. Nanosci. Nanotechnol. 15: 6397-6404. http://dx.doi.org/10.1166/jnn.2015.10736

Tan XH, Cheng R, Hu HP and Bai YP (2015). Classification of colon cancer based on the expression of randomly selected genes. Genet. Mol. Res. 14: 12628-12635. http://dx.doi.org/10.4238/2015.October.19.6

Xu XL, Yao YL, Xu WZ, Feng JG, et al. (2015). Correlation of MSH3 polymorphisms with response and survival in advanced non-small cell lung cancer patients treated with first-line platinum-based chemotherapy. Genet. Mol. Res. 14: 3525-3533. http://dx.doi.org/10.4238/2015.April.15.16

Genetics and Molecular Research 15 (3): gmr.15038388 